\title{
LA NUEVA TOMA DE DECISIONES EN EL MUNDO ACTUAL.
}

\section{THE NEW DECISION MAKING IN THE CURRENT WORLD.}

\author{
MSc. Francisco Raúl Arencibia Pardo, PhD. Saury José Thomas Manzano, \\ MSc. Belisario Peña Rodríguez.
}

Universidad de Pamplona, Ingenierías y Arquitectura, Ingeniería Industrial, Grupo de investigación (Investigación, Gestión y Administración de Producción y Operaciones) INGAPO

E-mail: \{francisco.arencibia, saurythomas, belisario.pena\}@unipamplona.edu.co

Resumen: El Presente trabajo muestra nuevas formas aplicadas a la hora de tomar decisiones. Herramientas utilizadas actualmente por el gerente empresarial frente a la incertidumbre, el riesgo y las turbulencias económico / sociales. Se presenta la aplicación de tecnologías de la información (TI) en la toma de decisiones, en la colaboración, el análisis y resultados. Diferentes soluciones unidas a la autonomía y capacidad de ostentar comportamientos por objetivos, tomando iniciativas cuando sea apropiado, interactividad y relación con el entorno, determinan que el uso de tecnologías de punta en la toma de decisiones, será el cercano futuro para el administrador.

La siguiente evaluación estadística: "el $81 \%$ de los consumidores esperan la misma experiencia de marca en todos los canales y el 54\% de los participantes en la encuesta pondría fin a su relación con un minorista si no se personaliza cada una de sus interacciones con él", es un perfecto reflejo del cambio.

Palabras clave Palabras claves: tecnologías de la información, toma de decisiones, dirección, colaboración.

\begin{abstract}
The present work shows new ways applied when making decisions. Tools currently used by the business manager in the face of uncertainty, risk and economic / social turbulence. The application of information technologies (IT) in decision-making, collaboration, analysis and results is presented. Different solutions linked to the autonomy and ability to show behaviors by objectives, taking initiatives when appropriate, interactivity and relationship with the environment, determine that the use of cutting edge technologies in decision making, will be the near future for the administrator.

The following statistical evaluation: "81\% of consumers expect the same brand experience in all channels and 54\% of the participants in the survey would end their relationship with a retailer if they do not personalize each of their interactions with he "is a perfect reflection of the change.
\end{abstract}

Keywords: technologies of the information, models, make decisions, managers. 


\section{INTRODUCCIÓN}

La nueva ola en el desarrollo informático está enfocada a operatorias de forma distribuida. No es un secreto los cambios en el entorno empresarial, por tanto, se hace impostergable la transformación en la concepción y diseño de estos sistemas. Por ejemplo, han aumentado las relaciones entre las organizaciones empresariales, se ha incrementado la necesidad de responder a un ambiente competitivo más dinámico y nos encontramos frente a un crecimiento exponencial de las empresas virtuales y de los negocios electrónicos.

Esto explicaría de un vistazo a qué nos enfrentamos:

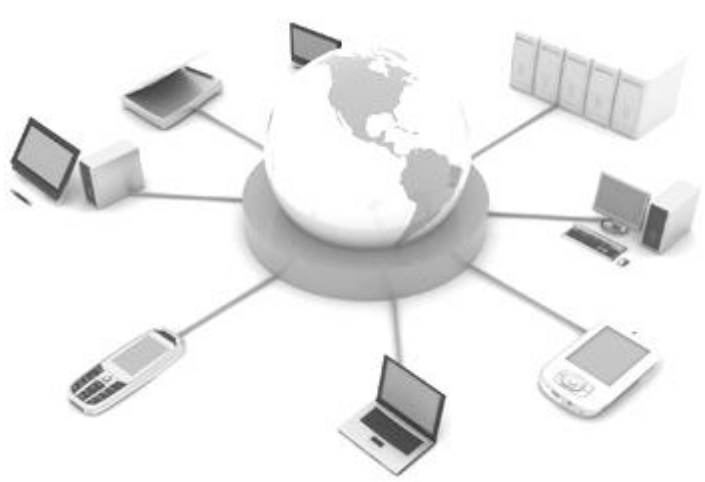

Fig 1. Setting up both Local Area Networks and Wide Area Networks for small and medium enterprises. Our solutions include consultancy, Planning, Designing, Implementation, Troubleshooting and Maintenance...

(fuente. http://www.iroclear.com/)

La época del ábaco, los libros contables y las decisiones tomadas "a dedo presencial" pasó a la historia (o prehistoria), sustituida por la generación de la información dirigida a todas y cada una de las alternativas posibles y sus consecuencias. Es la correcta recopilación de datos y su correcto tratamiento quien nos permitirá tomar decisiones convenientes. "Nos encontramos en una era de constante evolución - afirma el ingeniero Wayi Chaín Rocha -, tanto en el mercado como en la tecnología. Para sobrevivir, los administradores deben de estar en constantes cambios e implementarlos en sus empresas para mantenerlas en un nivel competitivo con un alto nivel de productividad. Todo esto, con un buen desempeño de las funciones administrativas y sometiéndose a un proceso de toma de decisiones continuo". Rocha, Wayi, 2006)

Es el turno del comercio sin presencia, de empresas en el éter, de negocios sin nada concreto de por medio. Es el momento de Netflix, Google y Amazon.

Ya en el 2013 se previó la denominada "nueva ola". Mónica Cordero nos comenta: personas conectadas con personas mediante dispositivos móviles. Personas que se comunican con dispositivos y máquinas conectadas con máquinas mediante censores. Se trata del "Internet de todas las cosas". Esta es la cuarta ola de Internet que ya está en proceso de desarrollo" ... (Cordero Sancho, Mónica, 2013)

Estos cambios, debido entre otros aspectos al desarrollo de la conectividad y las características de los entornos actuales, han significado una explosión de la disponibilidad de información y de recursos, un aumento en el uso y aplicación de las tecnologías en la actividad productiva y servicios, y una necesidad de integración entre las diferentes tecnologías.

Pero no todos los interesados en apoderarse de la alta tecnología y los soportes son gigantes multimillonarios. Hoy, miles de empresas pequeñas y medianas, pugnan en un mundo cambiante por tomar buenas decisiones, sobrevivir y desarrollarse. Nuestro entorno latinoamericano sufre como nunca el embate de gigantes comerciales bajo la capa de páginas web inocuas.

Miles de millones de dólares transitan hacia los Estados Unidos, China y otros a través de sitios digitales que ofrecen miles de productos de todo tipo, ninguno de los cuales fabrican.

Es el mercado y la lucha en el nuevo campo de batalla: la virtualidad.

Es el nuevo lema: el enfoque al cliente.

Es el nuevo reto: tomar malas decisiones ya no se perdona.

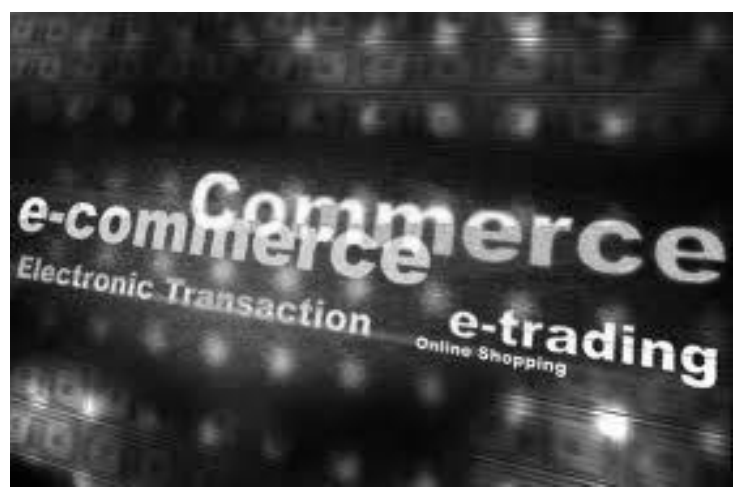

Fig 2. Diferencias entre una empresa virtual y una real. (Fuente. http://masterguapohacker.blogspot.com.co/2012/05/diferenciasentre-una-empresa-virtual-y.html)

Según estudio llevado a cabo por especialistas de la Universidad EAN en el 2015, solo el $20 \%$ de las Pymes colombianas sobrepasan el primer año de 
vida. Como causa principal a este descalabro plantean: "si hay algo en lo que están fallando las Pymes es en la poca importancia que le dan a la innovación y al conocimiento, los cuales deberían ser el centro y el corazón que hacen mover los otros ítems de importancia en las organizaciones".

(Pérez-Uribe, Rafael Ignacio, Ramírez, María del Pilar, 2015)

La pregunta a imponer es: ¿realmente estamos listos para asimilar el cambio?

El economista Santiago Escobar en su análisis sobre las características de la innovación empresarial que se tienen en cuenta en la política de transformación productora en Colombia, refleja las nuevas condiciones para el sector agrícola. (Escobar, Santiago A, 2012)

\section{Innovaciones tecnológicas en la agricultura moderna}

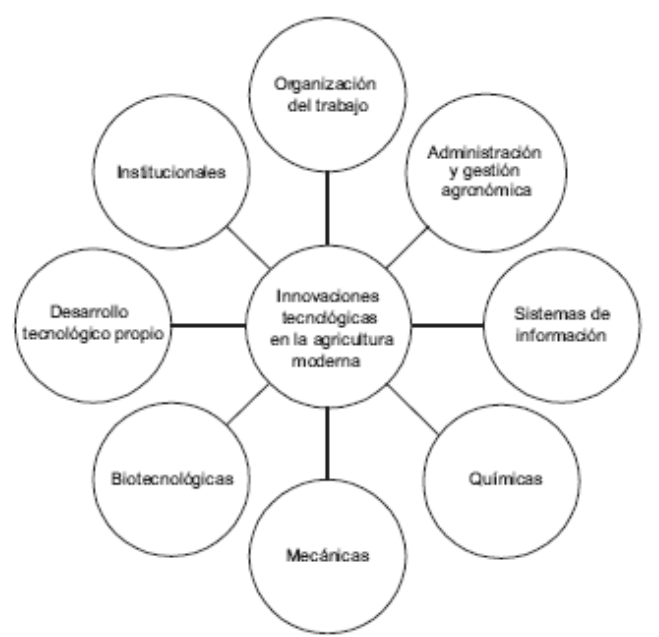

Fig 3. (Fuente http://www.scielo.org.ve/scielo)

Todos estos cambios causan acrecentamiento del nivel de complejidad en el ambiente empresarial y los negocios. Como consecuencia, se ha complejizado la toma de decisiones.

De forma clara, lo expresó Francisco Reyes: "pensar linealmente en un mundo que está regido por las tecnologías exponenciales, puede ser un problema para las organizaciones. Sí no cambiamos nuestro paradigma mental, ese pensamiento nos puede llevar al fracaso". (Reyes, Francisco, 2017)

Christian Fisher no es menos elocuente al enunciar: "A diferencia de las máquinas, las leyes o políticas, nuestras habilidades de toma de decisiones humanas pueden ser influenciadas por el mal estado de ánimo, fatiga, enfermedad, confusión, distracción y sesgo". (Fisher, Christian, 2016)
Como paliativo, se encuentran los sistemas basados en agentes, es decir, los agentes inteligentes y los Sistemas Multi-Agentes (SMA)

"Los enfoques tradicionales para gestionar los procesos de negocios a menudo son inadecuados para una gran escala, organización \pm amplia y configuración dinámica - apuntan los especialistas en inteligencia artificial Jennings, Norman y Faratin -. Sin embargo, dado que las tecnologías de Internet se han generalizado, un número creciente de procesos comerciales exhiben estas propiedades. Por lo tanto, se necesita un nuevo enfoque. Para este fin, describimos la motivación, la conceptualización, el diseño y la implementación de un nuevo sistema de gestión de procesos comerciales basado en agentes". (Jennings, T. J. Norman, P. Faratin Dept. Ingeniería Electrónica, Queen Mary \& Westfield College, Universidad de Londres, Londres, Reino Unido. 2000)

En un ambiente regido por la globalización, inflación y competitividad, los administradores de negocios, PYME, empresas y servicios, pueden mejorar la calidad y certeza en sus decisiones, trasladando la incertidumbre y los riesgos, utilizando TI a través de sistemas de soportes digitales, o DSS.

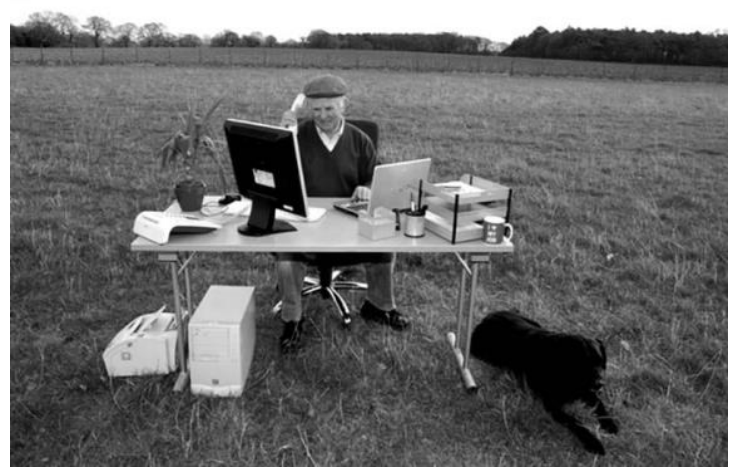

Fig 4. (Fuente https://www.adslzone.net/2015/05/04/

\section{LOS SISTEMAS DE SOPORTES DIGITALES.}

Un agente lo definimos como la entidad inteligente existente dentro de cierto contexto y que se comunica a través de mecanismos inter procesos, generalmente un sistema de red utilizando protocolos de comunicación 


\section{Tipos de Sistemas de Apoyo a las Decisiones}

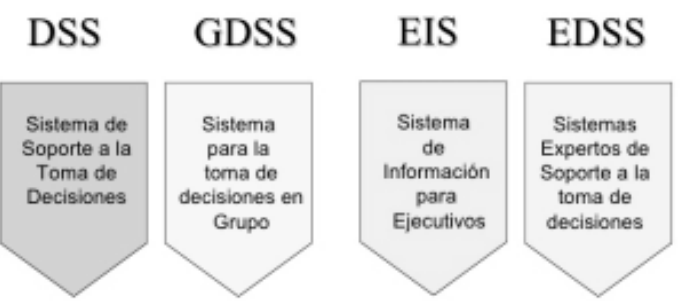

Fig. 5 Sistema de Soporte a las tomas de decisiones. (Fuente.http://sistemasdeinfo2016.blogspot.es/1466346519/siste mas-de-soporte-a-las-decisiones-dss-gdss-edss-eis/)

No obstante, la probada eficiencia de las TI, para una correcta toma de decisiones, estas deben encontrarse en interacción y entrelazadas con el dominio del problema. El gerente necesita una información precisa, incluso ser capaz de establecer pronósticos acertados, por tanto, las TI a través de las DSS deben ser activos en los procesos de decisión, capacitados en tomar iniciativas para ejecutar tareas relacionadas con las decisiones y hacer propuestas que permitan al usuario enfocarse en variantes ser significativas e importantes.

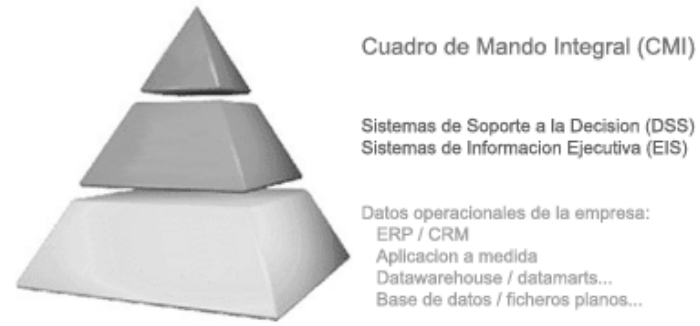

Fig 6. Cuadro de mando integral.

(Fuente. http://www.sinnexus.com/)

Para solo citar un ejemplo: Sedgwick James Inc. (Empresa de seguros más grande del mundo) desarrollo un DSS para manejo de riesgos para ayudar a minimizar las pérdidas de PepsiCo por accidentes, robos y otras. Como resultado, los gerentes de PepsiCo de todos los niveles pueden identificar con precisión tendencias críticas, indagar información de respaldo detallada, identificar problemas y plantear maneras de minimizar riesgos y maximizar utilidades.

Por tanto, la problemática específica es la premura en contar con sistemas de soporte a la decisión proactivos, relacionados con los usuarios, con el entorno y que tengan mayor flexibilidad en el acceso a la información. Para lograrlo hay que diseñar, mediante metodologías de desarrollo orientadas a agente, una arquitectura flexible en el acceso a la información, capaz de exhibir un comportamiento dirigido por objetivos, tomar iniciativas cuando sea preciso, relacionadas con su entorno a un DSS ya existente.

Los DSS, vistos de la forma más simple, se componen de:

- Un decisor (decision-maker): individuo o grupo encargados de tomar una decisión particular.

- Un conjunto de entradas al proceso de la toma de decisión (decision making): información de cualquier tipo según la situación específica, así como un conjunto de obligaciones y normas asociadas con la toma de decisión.

- Proceso de decisión: conjunto de pasos, modelos de decisiones, etcétera, que transforman las entradas en salidas en forma de decisión.

- Conjunto de salidas como consecuencias del proceso de decisión: serie de criterios para evaluar las alternativas de decisión, un conjunto de acciones a ejecutar o un conjunto de necesidades, problemas u objetivos a resolver.

Entre sus principios de diseño se encuentran:

La necesidad de lograr una comunicación interactiva con los decisores.

Flexibilidad en el acceso a la información para la toma de decisiones.

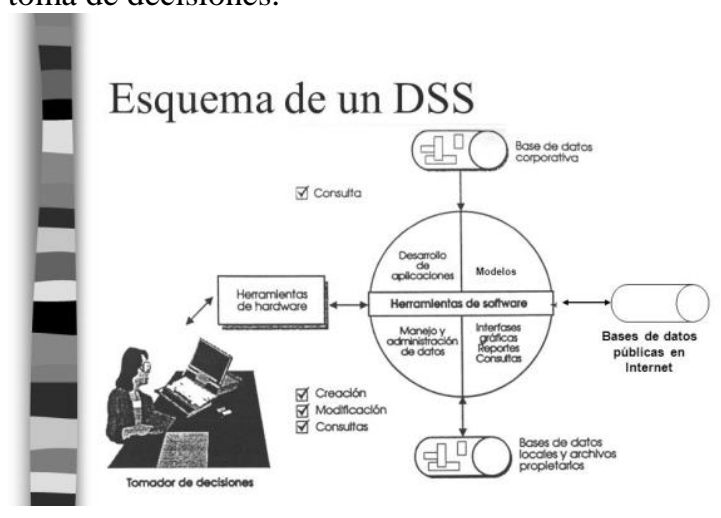

Fig 7. Interacción usuario sistema.

(Fuente. http://slideplayer.es/slide/13622308/)

Tomar decisiones un proceso complejo hasta para el mejor sistema pensante. Con este fin se han empleado distintas aplicaciones de la Inteligencia Artificial y tecnologías como los Data Warehouse y los Data Mining, desarrollando DSS de mayor calidad y modelando diversos aspectos dentro de la toma de decisiones. El progreso en la conectividad ha significado una explosión de la disponibilidad de información y de recursos, un incremento del uso de las tecnologías en los procesos de negocio y una necesidad de integración entre las tecnologías, 
lo que ha incrementado la complejidad en la toma de decisiones.

Los DSS actuales precisan amentar su nivel de proactividad, mantener una elevada y profunda relación con su entorno: El desarrollo de la conectividad demanda nuevos enfoques que permitan a los DSS ser parte de una infraestructura de información, a través de la interacción con su entorno. Esto le proporcionaría al sistema medios para percibir el entorno del problema y enriquecer sus capacidades cognitivas, así como para actuar sobre este para responder adecuadamente a las decisiones tomadas.

La relación de los DSS con su entorno es de gran importancia. El entorno de un DSS apoya la toma de decisión en diferentes formas:

- $\quad$ Preparación para la decisión. El entorno del DSS proporciona la información para el proceso de decisión, que puede provenir de las diferentes entidades de una organización y puede encontrarse en diferentes formas de almacenamiento. Además, permite informar al decisor de los cambios ocurridos en los procesos de negocio que puedan repercutir en la toma de decisión.

- $\quad$ Estructura de decisión. El entorno puede proporcionar herramientas o modelos para organizar la información de entrada, de manera que tenga sentido para el marco de la decisión.

- $\quad$ Proceso de decisión. El entorno puede automatizar parte del proceso de decisión y ofrecer evaluaciones o propuestas para la decisión óptima. Esto sugiere la necesidad de interoperabilidad e integración con otros sistemas, DSS u otros elementos para llevar a cabo el proceso de decisión.

- $\quad$ Propagación de la decisión finalmente tomada. El entorno sirve de vía para esparcir el resultado de la decisión por las dependencias o entidades interesadas.

Con el aumento de la interconexión, se requiere que estos sean expandidos e integrados con otros DSS o con diferentes fuentes de información. Los DSS aislados son un obstáculo a hora de tomar decisiones y son incompatibles con el hecho de que a cada momento puede aparecer información relevante. Necesitan ser parte de un ambiente integrado de información, que permita proporcionar a los clientes información relevante, responder a las situaciones de emergencia $y$ obtener y brindar información a los procesos de negocio.

Uno de los problemas que presentan los DSS desarrollados actualmente sin una relación estrecha con el entorno, está relacionado con el proceso de recuperación y actualización de información para la decisión, debido al aumento de la información disponible y al hecho de que esta pueda estar distribuida en diferentes entidades de la organización. Además, de nada vale que existan modelos de decisión muy eficientes si la información para la decisión no está actualizada o no está toda la que se necesita.

Por esta razón, y dada la importancia que tiene la información en un DSS, se plantea que para lograr un diseño más eficiente es preferible tener varios sistemas que permitan el acceso a la información dondequiera que se encuentre almacenada, en cualquier tipo y con diferentes modelos de almacenamiento, y ser capaz de identificar y conceder en tiempo información relevante que no haya sido explícitamente solicitada, lo cual se debe, en ocasiones al desconocimiento de su existencia.

\section{SISTEMAS BASADOS EN AGENTES PARA EL DISEÑO DE LAS DSS.}

Analizando las características requeridas de los DSS, así como las características de la organización y de los procesos de negocio, y, por otro lado, conociendo las potencialidades de utilizar sistemas basados en agentes (Checa Rojas Diego, Rojas Alvarado Oscar, 2014), se ha pensado en estos como una buena elección para el desarrollo de las DSS.

La selección de los Sistemas Multi agentes (SMA), como una solución tecnológica para modelar organizaciones, es motivada por:

- El dominio del problema implica una distribución inherente de datos, de información, de recursos y de posibilidades para resolver problemas (conforme al modelo básico de distribución y encapsulamiento de componentes en los sistemas basados en agentes para resolver problemas).

- Necesidad de mantener la integridad de la estructura organizacional existente y la autonomía de sus partes.

- Interacción entre las entidades de la organización, las que pueden ser altamente sofisticadas.

- La solución del problema no puede ser enteramente definida de antemano desde el principio hasta el final

- La dinámica de la estructura organizacional requiere que esta pueda ser modificada cuando proceda y esto puede ser logrado gracias al bajo acoplamiento que permite el paradigma de los agentes. 
En segundo lugar, la utilización de sistemas basados en agentes para el diseño de sistemas orientados a la toma de decisiones es una solución para construir DSS. En el caso de estos últimos, la utilización de agentes puede ser orientada entre otras actividades a:

- Buscar y utilizar fuentes de información, que puedan ser útiles para la toma de decisión, aunque estén distribuidas geográficamente.

- Permitir que esta información pueda ser integrada, actualizada y puesta a disposición de los decidores, y que este proceso les permita mejorar sus decisiones.

- Que la interactividad propia de los agentes proporcione, no solo un mecanismo flexible $\mathrm{y}$ adaptativo a los procesos de toma de decisiones, sobre todo en empresas virtuales y en sistemas de comercio electrónico, sino también la relación interactiva que se requiere con los usuarios decidores.

- Contribuir a que se automaticen más tareas para el usuario, y permitir que se requiera menos de su participación (proporcionando la interacción demandada entre el usuario experto y el sistema sin que este tenga el control de sistema en todo momento), y por tanto que la administración sea más indirecta.

- Generar alternativas de decisión y hacer propuestas que permitan al usuario enfocarse en variantes que pueden ser significativas.

- Estudiar el comportamiento de los usuarios a través de los perfiles y que permitan, de forma pro-activa, recomendar información relevante para los usuarios.

\section{ARQUITECTURAS PARA EL DISEÑO DE LAS DSS BASADOS EN AGENTES.}

La arquitectura de un software describe al sistema a un nivel macroscópico en términos de subsistemas, componentes, módulos, etcétera, así como la relación entre estos, a través de los datos que comparten y las dependencias entre ellos.

Las arquitecturas basadas en agentes siguen también estos principios, pero se centran en los aspectos relacionados específicamente con los agentes, cómo serán estructurados y organizados, las interacciones y la comunicación entre estos, sus roles y tareas dentro del sistema

Uno de los enfoques seguido para el diseño de arquitecturas o SMA en general, es el de analizarlos como estructuras organizacionales compuestas por agentes que interactúan y cooperan entre ellos para alcanzar un objetivo.
Las aplicaciones paralelas y distribuidas usualmente utilizan arquitecturas basadas en el principio de descomponer las funcionalidades y los datos requeridos por el sistema. Mientras en los sistemas multi-agentes, el comportamiento proactivo y autónomo de los agentes sugieren que las aplicaciones sean diseñadas imitando el comportamiento y la estructura de las organizaciones humanas, donde a cada agente se le asigne un rol determinado. La perspectiva organizacional permite que cada agente sea responsable de sus acciones para cumplir su rol, y reduce la distancia conceptual entre el sistema y el problema real que va a modelar y a resolver.

El diseño de arquitecturas para diferentes SMA, con uno $\mathrm{u}$ otro enfoque, tanto para problemas genéricos como para resolver problemas específicos en un dominio de aplicación han sido objeto de investigación. A continuación, se muestran distintos ejemplos de arquitecturas de agentes para desarrollar sistemas para la toma de decisiones. Estas fueron escogidas como ejemplo pues si bien todas utilizan las ventajas de esta tecnología, se enfocan o resaltan propiedades particulares en su diseño.

En el contexto de los sistemas abiertos y distribuidos existe una creciente necesidad de lograr sistemas situados en su entorno, es decir, que tengan una noción explicita del entorno en que se ejecutan y cómo las características de este pueden afectar sus funcionalidades.

La arquitectura tomada como ejemplo propone un enfoque para lograr DSS situados en su entorno. Está conformada por los elementos básicos de un SMA para relacionarse con este: sensores, efectores, así como una interfaz de usuario y un núcleo, como se muestra en la figura. Cada uno de estos componentes puede ser representado por agentes o SMA según el problema a resolver.

La información intercambiada entre los componentes del DSS y el usuario es indicada por D. $\mathrm{X}$ es la información obtenida del entorno, $\mathrm{Z}$ la información enviada al entorno y Y la información entre los componentes del DSS.

En principio, el papel de los sensores es recopilar, filtrar y procesar información relevante para el dominio del problema de varias fuentes de información, mientras, mediante los efectores el DSS, envía señales a su entorno para dirigir o alterar su estado. Pero tanto los sensores como los efectores no tienen que ser simples vehículos para la ejecución de las decisiones o la comunicación, sino que pueden incorporar mayores niveles de complejidad o inteligencia (Gelves Díaz John 
Freddy, Sánchez Molina, Jorge, 2012). Por ejemplo, pueden convertir la decisión en un conjunto de planes detallados, optimizar aspectos de la decisión, determinar la secuencia de las acciones, monitorear la ejecución de una decisión y conducir la negociación en el proceso de implementación de una decisión.

Entre las funcionalidades definidas para los sensores está:

planning: la más importante, pues permite la planificación, decide el orden y el momento en que las otras capacidades serán invocadas, qué fuentes de información serán solicitadas y utilizadas o cómo serán dirigidas las salidas.

conn: permite el acceso, por ejemplo, a las fuentes de información.

trans: filtra y procesa la información recogida.

alert: permite solicitar la atención inmediata del usuario o de otras partes del sistema.

adapt: da la posibilidad de adaptar el resto de las capacidades, en dependencia de la situación en que se encuentren.

Los efectores, por su parte, además del planning y el adapt, con una finalidad similar que, en el caso de los sensores, contiene:

conn: que en este caso es utilizado para ejecutar alguna acción que intente modificar el entorno, según la decisión obtenida.

trans: formula la acción a ejecutar para implementar la decisión generada por el DSS.

query: da la posibilidad de que, si los efectores necesitan información adicional de otros componentes para implementar una decisión, puedan solicitarla.

Su núcleo está compuesto por los elementos de un DSS tradicional, incluyendo modelos de decisión, bases de datos, y bases de conocimiento relevantes al dominio del problema. Incluye además un componente activo, el DSS administrador, que da la posibilidad al DSS de activarse o realizar determinadas tareas de forma autónoma, por ejemplo, contactar con el usuario, preparar al sistema para una interacción previa a la solicitud de un usuario e incluso tomar decisiones cuando el usuario no pueda ser contactado. Entre sus principales funcionalidades están:

generate: genera acciones de decisión directamente hacia los efectores.

query: permite solicitar información a los sensores. surgest: permite que el DSS sugiera comportamientos de forma pro-activa.

adapt y plan: similares a los efectores y a los sensores.
Interfaz de Usuario Activa: Los DSS requieren, particularmente, el establecimiento de una correlación entre el usuario y el sistema que permita mayor facilidad e interactividad en el diálogo. Uno de sus principales objetivos es mantener actualizado el perfil de usuario. Entre sus funcionalidades está:

conn: permite la comunicación con el usuario y con el sistema.

trans: permite transformar las entradas.

query: permite la solicitud de información requerida a otros componentes o al usuario.

alert, plan, adapt: con características similares a los sensores y a los efectores.

profile: que permite actualizar el perfil.

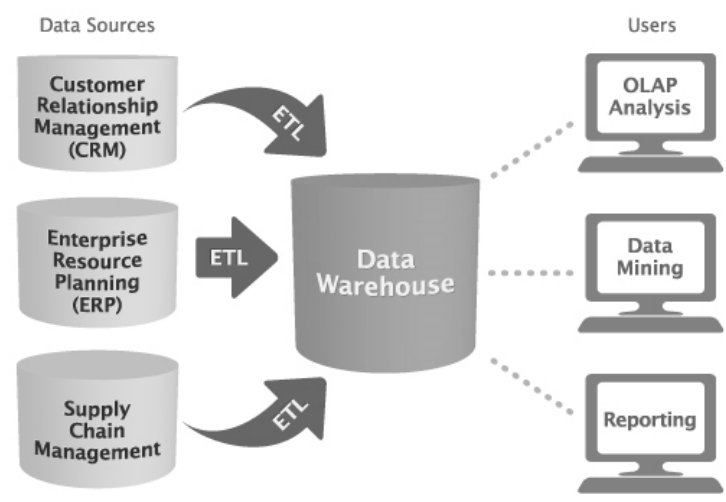

Fig 8. Data Warehouse. (Fuente. http://www.stratebi.com)

Es una realidad objetiva. Los sistemas de apoyo a la decisión se han vuelto populares. El presente anuncio, extraído de:

https://www.madrimasd.org/uploads/empresas/een/ documentos/boletin_een_mi+d_marzo17.pdf, es de hace muy poco tiempo.

EMPRESA GRIEGA BUSCA SOCIOS PARA DESARROLLAR CONJUNTAMENTE UN SISTEMA DE APOYO A LA DECISIÓN (DSS) BASADO EN GIS Y SENSORES REMOTOS CON APLICACIÓN EN MEDIOAMBIENTE (Ref. TRGR20170214001)

Una pyme griega especializada en sistemas de información geográfica basados en enfoques y equipos de alta tecnología busca socios tecnológicos con el fin de desarrollar y explotar conjuntamente un sistema de apoyo a la decisión (DSS) con aplicaciones en medioambiente. La empresa está equipada con una nueva infraestructura de monitorización aérea, específicamente con un avión ligero tripulado (LMA) y vehículos aéreos no tripulados (UAV) con distintos sensores para observación de la Tierra y grabación de parámetros ambientales críticos. La 
empresa busca compañías con experiencia en GIS y sensores remotos con el fin de establecer acuerdos de cooperación técnica, así como investigadores expertos en estas áreas para participar en proyectos de investigación.

\section{CONCLUSIONES.}

"Los ejecutivos hacen muchas cosas, además de tomar decisiones. Pero solo los ejecutivos adoptan decisiones. Por lo tanto, la primera actitud gerencial es el de tomar decisiones efectivas." (Drucker, 1989).

La virtualidad, los sistemas de apoyo a la toma de decisiones y el enfoque direccionado al cliente pisan fuerte $y$ no dan tregua. Las nuevas tecnologías de la información son útiles herramientas en la toma de decisiones. Cada vez y con mayor rigor, se utilizan para la mejora de la calidad en las disposiciones empresariales. El dilema estriba en que ninguna tecnología es capaz de analizar la totalidad de variables utilizando el factor subjetivo humano.

¿Resulta adecuado dejar nuestras decisiones a manos de tecnologías informáticas cada vez más poderosas y con mayor grado de autonomía?

... "pero las redes neurales pueden percibir correlaciones entre cientos de variables y pueden realizar muchas operaciones simultáneamente, reconocer problemas, generalizar, etc.

Los gerentes toman decisiones en grupos, sobre todo si son importantes, es por eso que es común verlos pasar gran parte de su tiempo en juntas definiendo problemas y buscando la manera de implementar las soluciones". (Mendoza, Andrés, 2014)

El tiempo dirá. Por el momento, resultan ventajosos pertrechos para los administradores de empresa y una ventaja competitiva.

\section{REFERENCIAS}

Acebal, César; Cueva, F.; Juan M. (2000). Acceso a bases de datos distribuidas mediante el uso de agentes móviles. Novatita, No. 146, Universidad de Oviedo. http://www.willydev.net/descargas/prev/dbmo viles.pdf

Arenas, Alvaro; Barrera, Gareth (2000). Applying the MAS-CommonKADS Methodology to the Flights Reservation Problem: Integrating Coordination and Expertise, Laboratorio de
Cómputo Especializado, Universidad Autónoma de Bucaramanga Colombia, 2000. http:// lpt.uni-mb.si/jckbse2002/aplication/ Apl_Login/upcamera/36-jckbse.pdf

Batzold, M.; Navarro, M.; Julian, V.; Botti V. (2003). Desarrollo de servicios turísticos a usuarios,

CAEPIA. http://grusma2.etse.urv.es/AgCitES/subpagine s/documents/f1.pdf

Booth, D.; Haas, H.; McCabe F.; Newcomer E.; Champion M.; Ferris, C.; D. Orchard (2004). Web Services Architecture, World-Wide-Web Consortium (W3C). Working Group Note. http://www.w3.org/TR/2003/WD-ws-arch20030808/

Bordini, R.; Braubach, L; Dastani, M.; Fallah, A.; Gomez, J.; Leite, j.; O’Hare,G.; Pokahr, A.; Ricci, A. (2006). A Survey of Programming Languages and Platforms for Multi-Agent Systems Informática 30 33-44.

Carlsson, C; Turban E. (2002). DSS: directions for the next decade, Decision Support Systems 33, 105-110.

Cordero Sancho, Mónica (2013). Viene una nueva ola informática, el financiero, https://www.elfinancierocr.com/tecnologia/vie ne-una-nueva-ola-

informatica/EZYNLIEWA5GRJIF6Q6CY7L $\mathrm{D} 2 \mathrm{XQ} /$ story/

De Roure, David (2005). Agents and the Grid - a personal view of the opportunity before us, Global Grid Forum Semantic Grid Research Group http://www.semanticgrid.org/documents/ agentgridlink/agentgridlink.pdf

Drucker, Peter F. (2007). The Essential Drucker: In One Volume the Best of Sixty Years of Peter Drucker's. (Butterworth-Heinemann.

Escobar, Santiago, Marleny Cardona, (2012). INNOVACIÓN EN LA TRANSFORMACIÓN PRODUCTIVA INDUSTRIAL: APORTES A LA DISCUSIÓN. Semestre Económico. Print version ISSN 0120-6346. Semest. Econ. vol. 15 no. 31 .

Espín, Rafael, Marx, Jorge, Hernández, Maritza, Lecich M.I. (2004). Logical management: fuzzy logic integrated models for decision making in enterprises, Trabajo presentado en World Automation Congress WAC.

Fisher, Christian. (2016). Los efectos de la mala toma de decisiones en el lugar de trabajo. https://pyme.lavoztx.com/los-efectos-de-la- 
mala-toma-de-decisiones-en-el-lugar-detrabajo-12308.html

Hernández-Vega José Isidro, Sanchez-Nigenda Romeo, Neira-TovarLeticia A. (2016). Sistemas MultiAgentes: Un Panorama de aplicación de distribución de ayuda en especie para logística humanitaria en situaciones postdesastres naturales. Daena: International Journal of Good Conscience. 11(1)156-167. ISSN 1870-557X

Hickman, F, Killin, J. Land, L., Mulhall, T, Porter, D. Taylor, (1989). R Analysis for KnowledgeBased Systems a practical guide to the KADS methodology, ESPRIT Project, University of Amsterdam.

Houmb, Siv Hilde, (2002). Security Issues in FIPA Agents, The Norwegian University of Science and Technology (NTNU), Norway. http://www.idi.ntnu.no/emner/ dif8914/essays/Houmb- essay2002.pdf

Jennings, N. R, Norman, T. Faratin, J., P.; Brien P. O' Odgers, B. (2000). Implementing a Business Process Management System using ADEPT: A Real-World Case Study. http://citeseer.ifi.unizh.ch/jennings00impleme nting.html

Mendoza, Andrés, (2014). ¿Cómo ayuda la tecnología en la toma de decisiones? https://prezi.com/3vjw2imhiesn/como-ayudala-tecnologia-en-la-toma-de-decisiones/

Pérez-Uribe, Rafael Ignacio, Ramírez, María del Pilar, (2015). Entrevista, ¿Por qué fracasan las pymes en Colombia? http://www.dinero.com/economia/articulo/py mes-colombia/212958

Reyes, Francisco. (2017). De la empresa lineal a la exponencial.

http://blogs.portafolio.co/negocios-en-laeconomia-digital/la-empresa-lineal-laexponencial/

Rocha, Wayi Chaín. (2006). DSS Sistemas de soporte a la toma de decisiones, https://www.gestiopolis.com/dss-sistemas-desoporte-a-la-toma-de-decisiones. 